\title{
The Elastic-plastic Deformation Analysis of the Hydraulic Pipe under Equivalent Static Loads
}

\author{
Runlian Jiang ${ }^{1,2}$, Yaohua Wang ${ }^{1}$,Chengfei Fan ${ }^{1}$ and Mianjun Duan ${ }^{1}$ \\ ${ }^{\prime}$ (College of Field Engineering, PLA Univ. of Sci. \&Tech, China) \\ ${ }^{2}$ (ZhenJiang Watercraft College of PLA, China)
}

\begin{abstract}
The finite element model of an aircraft hydraulic pipe was established using the ANSYS APDL command in this paper. The equivalent static pressures as surface loads were applied to the hydraulic pipe based on LS-Dyna software. The pressures were measured in the tire burst test. The stress, strain contours and time course curve of hydraulic pipe were obtained under the dynamic loads. The elastic-plastic deformations of the hydraulic pipe were analyzed by Von Mises yield criterion. The maximum stress is $1090000 \mathrm{KPa}$ beyond the yield limit. The hydraulic pipe occurred to plastic deformation. The maximum strain is 0.29168 . It is not exceed the elongation of the material. The pipe does not break.
\end{abstract}

Keywords: Aircraft tire burst jet; Equivalent static pressure; Hydraulic pipe; Elastic-plastic.

\section{Introduction}

The Aircraft tires have a high load capacity, high inflation pressure, a large heat, high-speed, continuous short distance glide and other characteristics, bursting by internal heat generation or foreign matter stabbing due to external pressure increases. The tire pressure inside the aircraft tire is $1.73 \mathrm{MPa}$ at the normal working hours, so it is compared to the "rubber bomb". Accidental tire burst will produce some powerful impact jets, they will threat airframe, engine and fuel tank safety. The equipments, pipes in the aircraft main landing gear compartment will be the impact of blasting air powerful of dynamic loads.

When the aircraft main wheel grounded, the spoilers ejected to damage wing lift, which achieved the purpose of deceleration speed. They playing important roles during the aircraft landing. The spoiler is packed up and ejected by hydraulic power. According to a predetermined the spoiler packs up and ejects, which is a reliable guarantee for safe landing. Once the tire burst, the resulting strong jet spoiler will crate a powerful impact on hydraulic pipe because the high pressure gas releasing inside the tire instantaneous. Hydraulic pipe will occur to elastic-plastic deformations under dynamic loads. Ensuring the normal aircraft landing, it is very important to research the elastic-plastic deformations of the hydraulic pipe under the impact of the aircraft tire burst jets. It is the premise to determine whether the hydraulic piping plastic deformation or breaking.

The equivalent static pressures as surface loads were applied to the hydraulic pipe based on LS-Dyna software in the paper. The tire burst modes are clearly defined in JAA standards. The characteristics of stress and strain of hydraulic pipe were studied under the dynamic loads[1]. The maximum strain and stress of hydraulic pipe were obtained at different times. The results determine whether the pipe produced plastic deformation or fracture damage. These provide bases for design and manufacture of the hydraulic pipe.

\subsection{Unit Type and Material Model}

\section{Building Model}

The model can objectively reflect the main structural characteristics of the hydraulic pipe in the aircraft main landing gear compartment and meet the dynamic strength analysis of the hydraulic pipe. Unit type elements used SHELL because of it being thin-walled pipe[2]. Thinking of the safety of the aircraft hydraulic lines, once plastic deformation of the hydraulic pipe took place, it is considered exceeding the aircraft safety and need to replace. Bilinear material model used in the analysis[3]. Hydraulic pipe used in the mechanical properties of metallic materials are listed in Table 1[4].

Table 1 Material mechanical properties of the hydraulic pipe

\begin{tabular}{|c|c|c|c|c|c|c|}
\hline $\begin{array}{c}\text { Material } \\
\text { Name }\end{array}$ & $\begin{array}{c}\text { Density } \\
{\left[\mathrm{kg} / \mathrm{m}^{3}\right]}\end{array}$ & $\begin{array}{c}\text { Elastic Modulus } \\
{[\mathrm{kPa}]}\end{array}$ & $\begin{array}{c}\text { Poisson } \\
\text { ratio }\end{array}$ & $\begin{array}{c}\text { Yield stress } \\
{[\mathrm{kPa}]}\end{array}$ & $\begin{array}{c}\text { Tangent modulus } \\
{[\mathrm{kPa}]}\end{array}$ & Elongation \\
\hline $21-6-9$ & 7860 & $2.0 \times 10^{8}$ & 0.28 & $7.45 \times 10^{5}$ & $1.8 \times 10^{6}$ & $56 \%$ \\
\hline
\end{tabular}

\subsection{Finite Element Model}

Hydraulic pipe is tubular model, a hollow tube, and outer diameter of D is $6.335 \mathrm{~mm}$, diameter $\mathrm{d}$ is $5.54 \mathrm{~mm}$. Finite element model was established by ANSYS APDL command modes. First Using shell181 
implicit elements do pre-stress analysis. Total unit 3000 and node 3020 generated. The finite element model of the hydraulic pipe is shown in Figure 1.

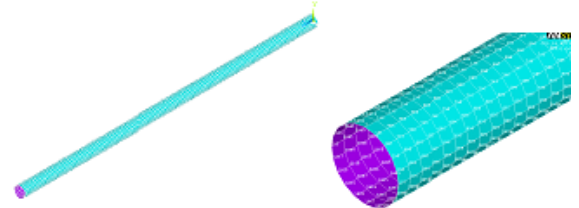

Fig. 1 Finite element model of the hydraulic pipe

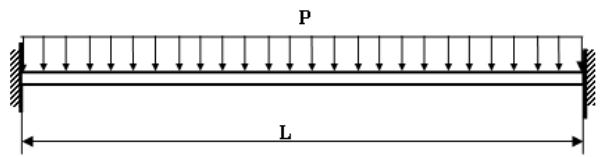

Fig. 2 The hydraulic pipe stress schematic

\subsection{Boundary Conditions}

\section{Loading and Boundary Conditions}

Hydraulic system pipe was used to transfer a working fluid medium having a certain pressure. The hydraulic pipe was suffered from fluid pressure and weight effects. The pipe was fixed with clamps at both ends. Pipe constraints can be assumed to be fixed constraint. Constrained distance L is $352.4 \mathrm{~mm}$. The tire burst over pressure P may be assumed to be uniformly distributed on it. As shown in Figure 2.

The liquid in pipe was handled with additional mass in the paper. The role of liquid was equivalent to the mass attached to the structure. Fluid was considered to incompressible and static. Only the additional mass related to the shape and structure. In the analysis, the first solution was initial stress using ANSYS implicit. Before the explicit dynamic analysis they were added to the structure, included in the explicit analysis in using LS-DYNA for structural dynamic response. Density of the liquid in the hydraulic pipe is $1000.8 \mathrm{~kg} / \mathrm{m}^{3}$, pressure is $20685 \mathrm{kPa}$.

\subsection{Load Applied}

Powerful impact airflow formed when the tire burst, and they is applied to the hydraulic pipe in the form of impact load with no considering the thermal stress in tire burst. The distance is $318 \mathrm{~mm}$ from the Hydraulic pipe to the spout.

The blast wave was loaded into the pipe with the forms of the equivalent static loads on analysis the elastic-plastic deformations of the hydraulic pipe. Considering the bearing area is far smaller than the conduit jet distribution, it assumed that the equivalent static pressures are equal on the entire surface of the pipe everywhere.Equivalent static bursting process is equivalent to the average pressure at the sensor position, calculated using the formula: $\frac{1}{\Delta t} \int_{0}^{0.02} P(t) d t$

Taking into account the tire burst process continued for about $10 \mathrm{~ms}$, tire pressure is reduced to $5 \%$ of the Initial inflation pressure. Based on the test data, the equivalent static pressure was calculated, the equivalent static pressure is $0.63 \mathrm{MPa}$. Equivalent static pressure loads curve diagram is shown in Figure 3.

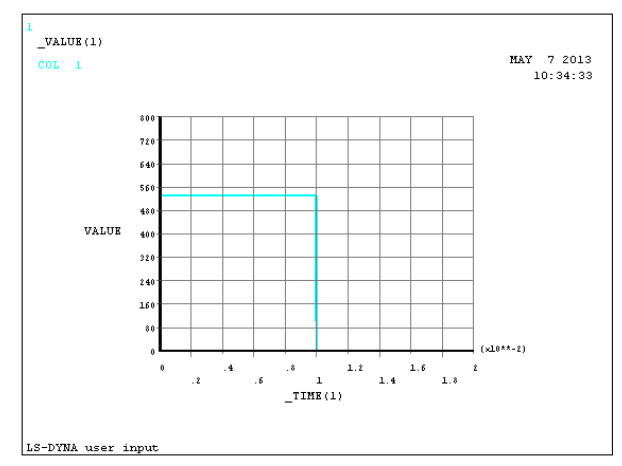

Fig. 3 Equivalent static pressure loads curve diagram

\subsection{The Von. Mises yield criterion}

\section{Calculation and Analysis of Results}

It is that the point begin to enter the plastic state when the point of the bearer object equivalent stress reaches a certain value (material allowable stress) to the Von. Mises yield criterion under certain deformation conditions. The physical meaning is refers to the material produce yield deformations under certain deformation conditions, per unit volume of the elastic potential energy (known as elastic deformation energy) reaches a certain constant when the material shape changes. 
By the Von. Mises yield criterion, the equivalent stress is:

$$
\bar{\sigma}=\frac{1}{\sqrt{2}} \sqrt{\left(\sigma_{x}-\sigma_{y}\right)^{2}+\left(\sigma_{y}-\sigma_{z}\right)^{2}+\left(\sigma_{z}-\sigma_{x}\right)^{2}+6\left(\tau_{x y}^{2}+\tau_{y z}^{2}+\tau_{z x}^{2}\right)}
$$

4.2 Analysis of stress, strain under the equivalent static pressures

It shows that the time is $10 \mathrm{~ms}$ when tire pressure released to $5 \%$ by the aircraft tire burst test data. The loads loading time extended to $19 \mathrm{~ms}$ because of the material strain hysteresis under dynamic pressure.

The von Mises stress and strain distributions of the hydraulic lines under equivalent static loads corresponding to two moments are shown in Figure 4. In the beginning of loading, there is an initial pre-stress because of liquid inside piping, at the dynamic loading $1 \mathrm{~ms}$, stress of the pipe fixed end produced mutation, which reached $963065 \mathrm{kPa}$ beyond the yield limit, at this time the pipe produced plastic deformation. At the time the elastic tubing, plastic deformation occurred simultaneously. With the increase of the loading time, the middle pipe stress gradually increased.The stress reaches a maximum at $11 \mathrm{~ms}$. The von Mises strain cloud charts are shown in Figure 5. The material deformation became slowly[5], it was time for 5ms to load with the dynamic loads, the strain reached maximum which is 0.29168 .
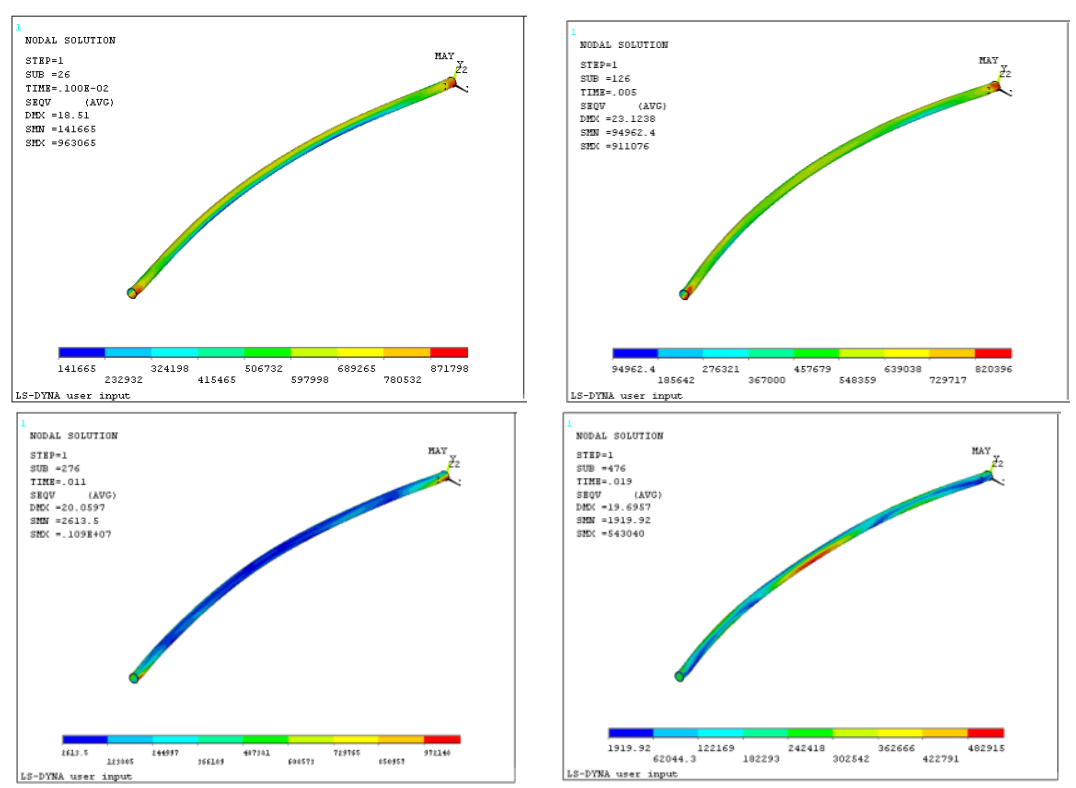

Fig. 4 von Mises stress distribution under the equivalent static loads
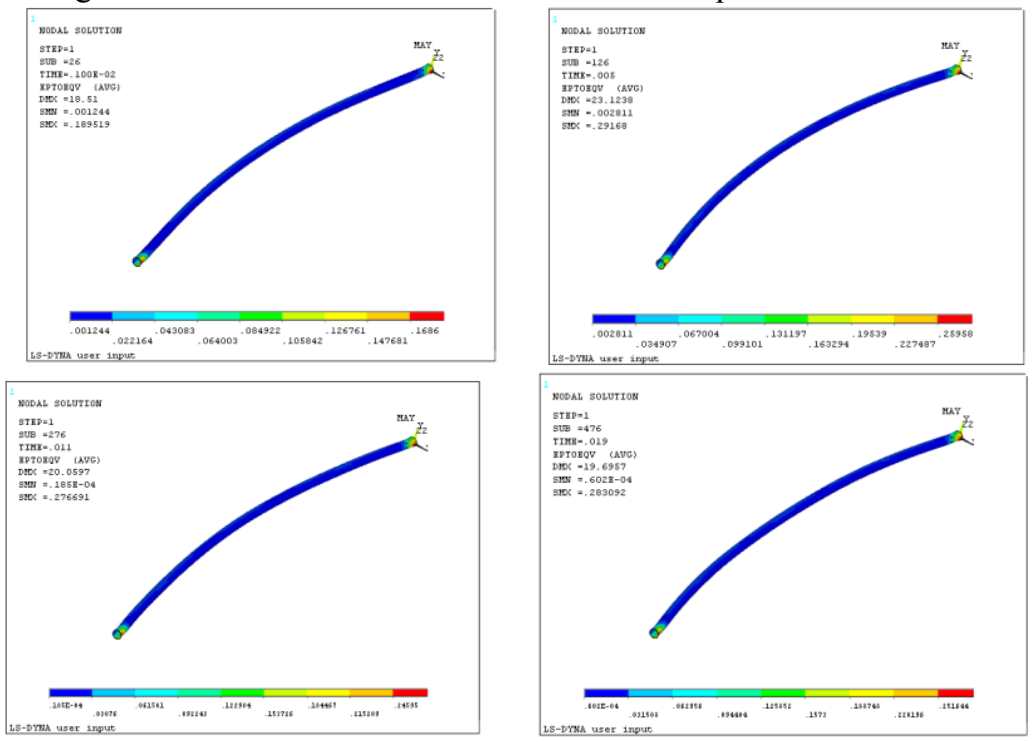

Fig. 5 von Mises strain distribution under the equivalent static loads 


\subsection{The process analysis of change in Stress and strain}

Table 2 shows the maximum von Mises stress and strain values of the hydraulic pipe under the equivalent static loads when every $1 \mathrm{~ms}$. From the table, the maximum stress has exceeded the yield limit, the maximum strain values were less than the elongation. Thus the plastic deformations occur to the hydraulic pipe under such load in some time, but do not break. The maximum strain and stress does not occur at the same time. The maximum strain always appears after maximum stress due to the characteristics of material delayed deformation. This conclusion can also be reached from the stress and strain contours. Since the selection of elastic-plastic material model, the elastic and plastic deformations occur simultaneously, the stress and strain are no longer simple linearity.

Table 2 The maximum stress and strain of the pipe under the equivalent static pressures

\begin{tabular}{|c|c|c|}
\hline Time $(\mathrm{ms})$ & The maximum stress $(\mathrm{kPa})$ & The maximum strain \\
\hline 1 & 963065 & 0.189515 \\
\hline 3 & 461030 & 0.283665 \\
\hline 5 & 911076 & 0.29168 \\
\hline 7 & 894099 & 0.291329 \\
\hline 9 & 430744 & 0.285706 \\
\hline 11 & 1090000 & 0.276691 \\
\hline 13 & 637689 & 0.27873 \\
\hline 15 & 378670 & 0.280911 \\
\hline 17 & 364496 & 0.283166 \\
\hline 19 & 543040 & 0.283092 \\
\hline
\end{tabular}

Take the stress distribution diagram of the pipe fixed end for example, the relationship between stress, elastic strain, plastic strain and time are respectively, shown in figure 6,7,8 and 9.

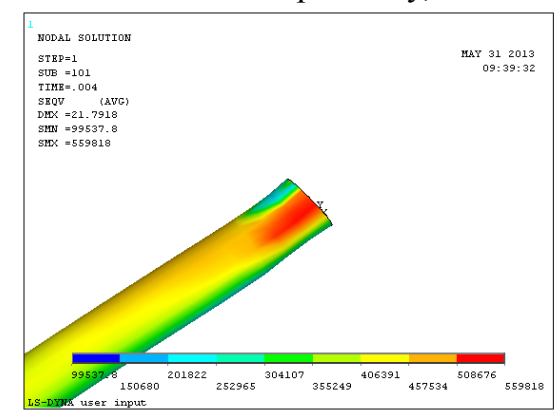

Fig. 6 Stress distribution of the pipe end

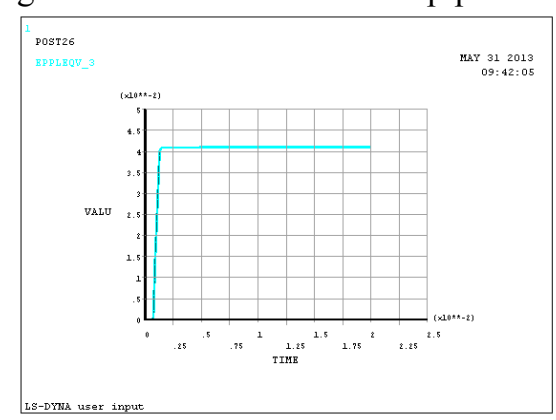

Fig. 8 Relationship between plastic strain and time

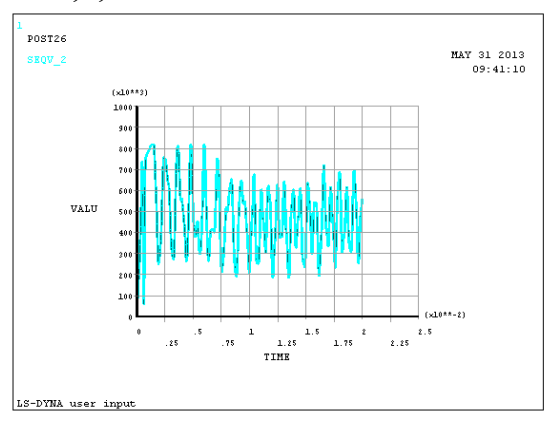

Fig. 7 Relationship between stress and time

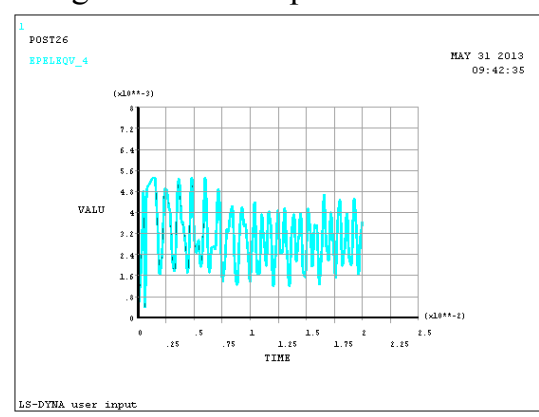

Fig.9 Relationship between elastic strain and time

The stress of the pipe fixed end is Maximum when the loading time is $4 \mathrm{~ms}$ from the figure 6.The maximum is $559818 \mathrm{kPa}$. Because the material is elastic- plastic model, the elastic deformation exists in the whole loading process, but the plastic deformation appears when the stress is over the yield limit at the loading time $0.6 \mathrm{~ms}$. There are strict similarity relation between stress and elastic strain from the figure 9 . There are three stages in the initial elastic strain, respectively is the approximate linear rising stage, gently rising stage and rapid decline stage. The strain changes with the stresses after the elastic strain dropped to the minimum value of the initial strain. Some scholars have studied the relations between the elastic strain and plastic strain which are the decomposition of plastic deformation [6]. The first and second phase boundaries in the initial elastic strain, plastic deformation occurs, rises rapidly with linear, retains transitorily and ascends slightly until keeping phase in full time course. The plastic strain remains unchanging after it reaches the maximum due to external loads are pulses unchanging with time. 


\section{Conclusions}

1) From the stress and strain cloud charts, since the presence of liquid in the pipe, the initial stress is greatest at the pipe fixed end, so the strain exists only at the fixed end of the pipe.

2) Under equivalent static pressure is $874698 \mathrm{KPa}$, it beyond the yield stress state. The maximum strain reach 0.180546 , but the elongation strain values are less than $56 \%$. Therefore, the pipe is not broken under the kinds of loads.

3) From the relationship between stress, elastic strain, plastic strain and time, the elastic strain reaches the maximum while the stress reaches the maximum. The plastic strain remains unchanging after it reaches the maximum because of lagging.

\section{References}

[1] Liangyong Gu and Jingwu He, Research of Dynamics Intensity on Planes'Structure, Aircraft Design, 29 (2), 2009,29-31

[2] Tao He, Jing Yang and Xing Jin, ANSYS 10.0/LS-DYNA Nonlinear Finite Element Analysis Instances Tutorial.(BeiJing :China Machine Press, 2007).

[3] Jihong Bi, Hui Wang, elastic-plastic engineering mechanical.(Tianjing :Tianjing University Press,2003).

[4] Qinghua Du. Engineering Mechanics Handbook.(Beijing: Higher Education Press, 1994).

[5] Jian Zhang, Research on Issues of Elastic-plastic Dynamic Time-history Analysis[J].Earthquake Resistant Engineering and Retrofitting 2011,33(5):74-78.

[6] You Li, Zhida Chen, Weishen Zhu, Research on relationship between elastic and plastic deformation of material[J]. Rock and Soil Mechanics, 1988, 9(3): 41-50. 